\title{
Synthesis of Modelling and Simulation for Hydrogen Gas Release and Explosion
}

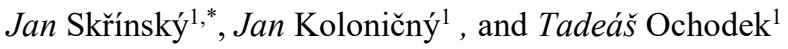 \\ ${ }^{1}$ Energy Research Centre, VSB-TU Ostrava, 70833 Ostrava - Poruba, Czech Republic
}

\begin{abstract}
The article presents the results of two methods for modelling and simulation of the leakage, dispersion and explosion of hydrogen in the event of major accidents in urban areas. Modelling of flammable substances by standard methods fully based on input data. Therefore, the predictions from common software packages should be extended to experimental results. The aim of this paper is to present the synthesis of modelling and simulation for one component gas-air dispersion and explosion to demonstrate the rational approach to loss prevention based on understanding of the nature of incidents and of the type of loss that actually occur.
\end{abstract}

\section{Introduction}

\subsection{Interest}

In the present article we obtained a set of experimental data needed to evaluate the explosion severity of hydrogen using a combination of experimental methods and suitable standard software simulation procedures. Consequence evaluation is an important part in risk assessment even if the results often contain many uncertainties. A method to identify and quantitatively evaluate such uncertainties would be to carry out a comparative study of experimental models and CFD models [1]. Selected application cases include the solution of current research tasks related to fundamental aspects of the effective syngas cleaning with the aim of increasing the hydrogen gas component [2].

\subsection{Previous studies}

Modelling the consequences of accidents in the Czech Republic has a long tradition among safety-oriented fields [3]. This research is located in the analytical field of industrial safety. The article offers a unique opportunity to present results of modelling with world-renowned computing models for predicting emergency situations. In fact, the data obtained can be used by professionals in the fields of fire prevention, explosions and toxic releases, but also in risk analysis and prevention. The results will help to orientate and avoid quickly mistakes in accepting absurd, overly conservative or overly optimistic results [4].

\footnotetext{
* Corresponding author: jan.skrinsky@,vsb.cz
} 


\section{Experimental model}

\subsection{High-speed camera}

The high-speed camera Photon FASTCAM SA-Z (speed $>1 \mathrm{M}$ fps; resolution $>12 \mathrm{k}$ fps) has been used to capture high-resolution digital images at ultra-high speeds. The high-speed camera has been installed in a chamber opening and focused on ignition source. FASTCAM Viewer tools allow image calibration and measurement of angles and distances from explosion image data [5].

\subsection{Experimental Setup}

The experimental setup used for determination of hydrogen-air explosion limits is schematically described in described in [5].

\subsection{Experimental procedure}

Lower explosion limits of the hydrogen-air mixture were determined experimentally according to the [6]. Procedure for gas explosion starts with evacuation to leave a space for the hydrogen gas. For example, the use of hydrogen concentrations of $30 \mathrm{vol} \%$ and 35 vol. \% require the partial pressures of hydrogen to be added are 0.30 bar and 0.35 bar respectively. Then the evacuation pressures should be 0.70 bar and 0.75 bar respectively. PLC starts the experiment where $600 \mathrm{~ms}$ is counted as ignition delay time, and then the gas is mixed by blowing dispersion air into the hydrogen-air mixture inside the chamber. After that the mixture is ignited by the electric discharge. The explosion characteristics are measured and calculated [7].

\subsection{Calculation procedure}

The explosion pressure in a constant volume chamber have been simulated based on the adiabatic assumption through thermal equilibrium. The element potential approach in the thermochemical equilibrium calculations applied in the Chemkin subroutine has been used for explosion pressure calculations. This approach represents "ideal" deflagrations in closed systems well and gives the highest possible attainable explosion pressures. Explosion pressures are calculated using the species and their thermodynamic values from the GRI 3.0. The mechanism used for the simulation was developed by Burcat. For the calculation we used Chemical Equilibrium Calculator [8].

\section{SW simulation}

\subsection{Simulation setup}

Areal Locations of Hazardous Atmospheres is the hazard modelling program for the CAMEO ${ }^{\circledR}$ software suite, which is used widely to plan for and respond to chemical emergencies. Threat zones can also be shown in Google Earth using KML export feature [8]. The SW package represents well-known pre-accident modelling tools commonly employed in assessment of dangerous substances releases, dispersion, and other effects and consequences [1]. 


\subsection{Simulation procedure}

The distances of hazardous zones were taken under the worst condition from the point of view of cloud dispersion weather conditions (stability class $\mathrm{F}$ and $2 \mathrm{~m} . \mathrm{s}^{-1}$ ). Volume of the vessel was 1000-L. Material characteristics were taken from updated chemical library, including new DIPPR chemical data. Figure 1 illustrates the threat zone estimates on a grid in used SW. A threat zone is an area where a hazard such as ignition concentration, thermal radiation or overpressure have exceeded a user-specified Level of Concern (LOC).

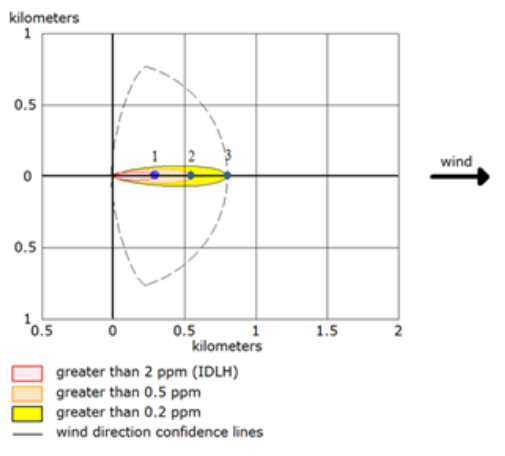

a)

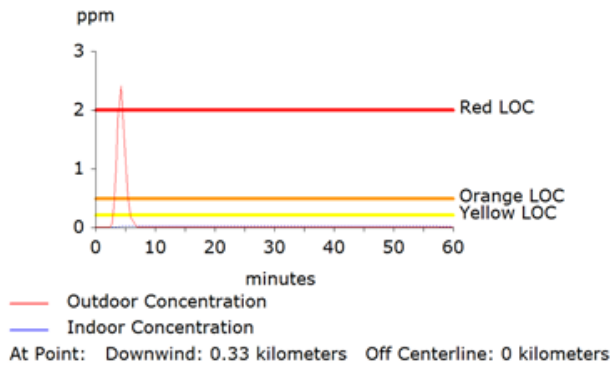

b)

Fig. 1ab. Threat Zones for $99 \%$ (red), $50 \%$ (orange) and 1\% (yellow) criterion.

\section{Results of modelling and simulation}

\subsection{High-speed images}

Figure 2 illustrates the turbulent spherical flame that have been produced in the concentrations close to lower explosion limit.

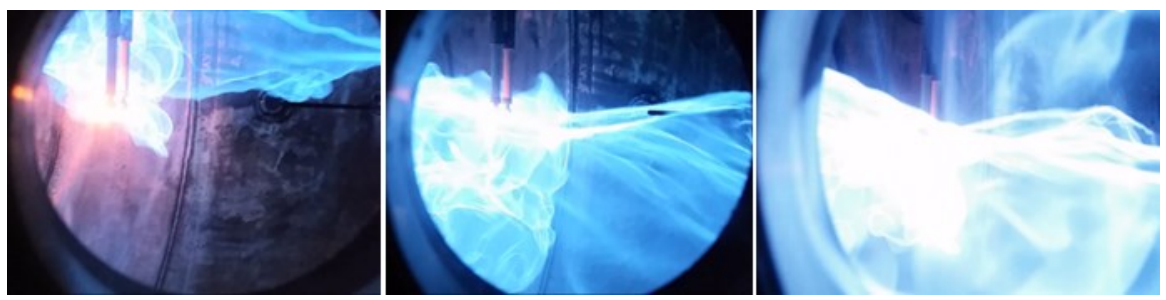

Fig. 2. High-speed images of hydrogen-air mixture close to lower explosion limit.

\subsection{Explosion parameters}

Figure 3 illustrates the explosion pressure $\left(P_{e x}\right)$ versus hydrogen concentration $(C)$ at initial atmospheric pressure $1 \mathrm{bar}$ and temperature $25^{\circ} \mathrm{C}$. The maximum explosion pressure $\left(P_{\max }\right)$ and maximum adiabatic explosion pressure $\left(P_{a d, \max }\right)$ give its peak values between $\mathrm{C}=30-35$ vol.\%. The difference between $P_{\max }$ and $P_{a d, \max }$ is remarkably increased at highly rich mixtures. This is due to the absence of oxygen and the heat loss through the continuum radiation to the vessel wall. 


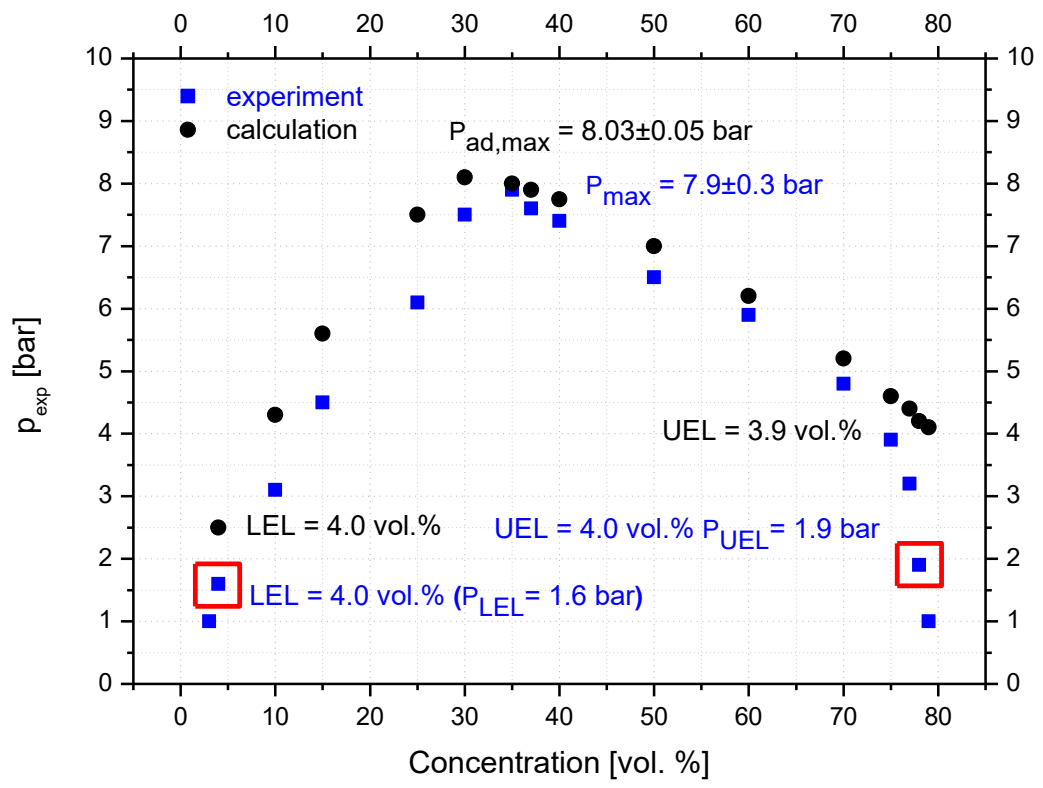

Fig. 3. Explosion pressure versus fuel fraction.

The experimentally obtained LEL $=4.0$ vol.\% $\left(P_{L E L}=1.6\right.$ bar $)$ have been implemented as the LOC input for SW simulation. The results of SW simulation are therefore supported by real experimental measurement with the knowledge of uncertainties and setup use. This introduces general methodological advantage.

\subsection{Numerical simulation}

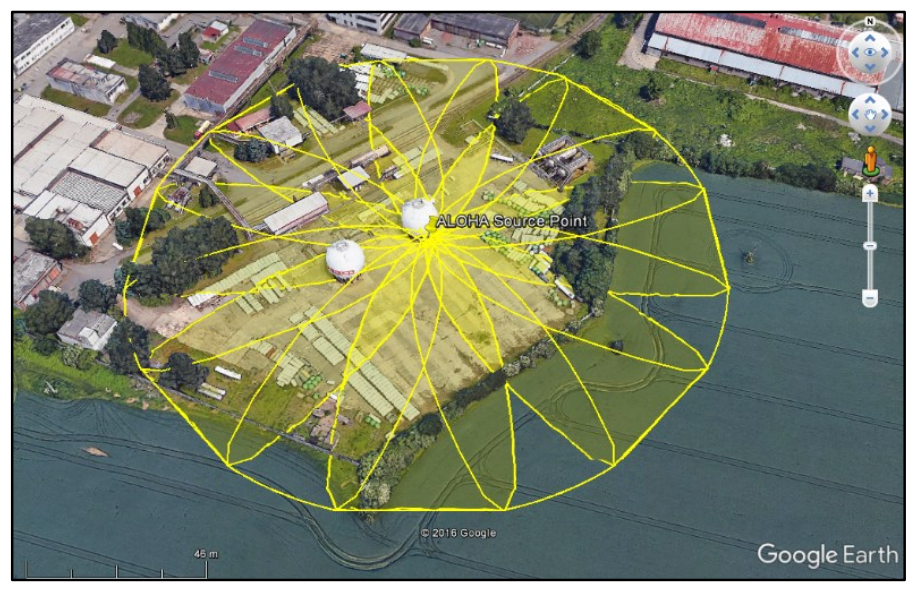

Fig. 4. Results of accident release of hydrogen implemented in Google Earth.

The results of hydrogen leak and dispersion have been implemented in Google Earth and presented in Figure 4. The yellow threat zone represents the ignitable concentration level. 


\section{Conclusion}

In the presented article we presented the methodological synthesis of experimental physical modelling and numeric simulation for hydrogen gas release. First, the explosion limits are investigated by both the 1000-L explosion chamber and chemical equilibrium calculations. Second, the measured and calculated results are implemented as the LOC input for SW simulation of hydrogen leak from real hydrogen storage technology. Combining the SW simulation and experimental modelling for investigation of gas dispersion offers great possibilities for further CFD support and simulations development. This work started a systematic investigation of hydrogen gas behavior that combine heated 1000-L explosion apparatus available at Energy Research Centre, VŠB - Technical University of Ostrava with well-known pre-accident modelling tools.

This study was carried out as part of the project: 'Research of the conversion of fuels to hydrogen and the safety of hydrogen technologies' (identification code SP2020/111).

\section{References}

1. A. Bernatik, W. Zimmerman, M. Pitt et al. Process safety and environment protection 2008, 86, 198-207

2. T. Ochodek, J. Koloničný, J. Skřínský et al. Ostrava: Report n. SP2020/111

3. M. Skř́nská et al. Materials Science Forum 2015, 811, 91-94

4. M. Skřínská et al. WSEAS Transactions on Environment and Development 2014, 9, 243250

5. J. Skrinsky, J. Koloničný, T. Ochodek MATEC Web of Conferences 2018, 168, 07013

6. EN 1839: Determination of the Explosion Limits and the Limiting Oxygen Concentration (LOC) for Flammable Gases and Vapors; European Committee for Standardization: Brussels, Belgium, 2017

7. ALOHA, 2020, CAMEO ALOHA, U.S. EPA, downloading from http://www.epa. gov/ceppo/cameo/aloha.htm

8. J. Skrinsky, MATEC Web of Conferences 2018, 168, 06006 